\title{
Hyper-frontality in an OCD patient - evidence from event-related potentials in a cued GO/NOGO task
}

\author{
Jolanta Zielińska', Jolanta Góral-Półrola² ${ }^{2}$ Paweł Półrola ${ }^{3}$, Maria Łuckośs ${ }^{4}$ Juri D. Kropotov \\ Maria Pąchalska ${ }^{4,7}$ \\ ${ }^{1}$ Department of Special Pedagogy, Pedagogical University, Kraków, Poland \\ ${ }^{2}$ Old Polish University, Kielce, Poland \\ 3 Jan Kochanowski University, Kielce, Poland \\ ${ }^{4}$ Chair of Neuropsychology, Andrzej Frycz Modrzewski University, Kraków, Poland \\ ${ }^{6}$ Laboratory for the Neurobiology of Action Programming, Institute of the Human Brain, Russian Academy of Sciences, \\ St. Petersburg, Russia \\ ${ }^{7}$ Center for Cognition and Communication, New York, NY, USA
}

Zielińska J, Góral-Półrola J, Półrola P, Łuckoś M, Kropotov JD, Pąchalska M. Hyper-frontality in an OCD patient - evidence from event-related potentials in a cued GO/NOGO task. Ann Agric Environ Med. 2016; 23(2): 276-279. doi: 10.5604/12321966.1203890

\begin{abstract}
Objective. The study aimed to evaluate the hyperactivity of the frontal lobes in a patient with obsessive-compulsive disorders.

Case description. A 21-year-old female met the diagnostic criteria of DSM-5 since her behaviour was characterized by a preoccupations with negative thoughts and by repetitive attempts to decrease or stop those behaviours. Two working hypotheses were tested to find neuromarkers of OCD and anxiety in the patient described. In agreement with the 'OCD hypothesis' an increase of the frontal beta activity and an increase of the parietalalpha activity pattern was found. The 'anxiety' hypothesis found confirmation in an increased left temporal P1 wave in response to the visual stimuli observed in ERPs. In all three conditions (EO, EC, GO/NOGO task), two characteristics were deviant from the normative average data in EEG spectra. First, an increase of frontal beta activity and the increase of parietal alpha activity was noted. The independent component analysis applied for 700 ms EEG fragments in GO and NOGO conditions revealed a strong activation over the central areas.

Conclusions. Specific patterns of QEEG and ERPs, the increase of beta activity frontally, and the increase of parietal alpha activity pattern which produce hyper-frontality, might be useful in the diagnosis of an OCD patient. ERPs in a GO/NOGO task can be used in the assessment of functional brain changes in OCD patients.
\end{abstract}

\section{Key words}

repetitive behaviours, neuromarkers, frontal beta activity, parietalalpha activity

\section{INTRODUCTION}

The term obsessive-compulsive disorder (OCD) emphasizes the nature of symptoms of this disorder, which are characterized by repetitive thoughts and behaviour. The disorder is prolonged to adulthood in contradistinction to fixation on a particular activity or ritual actions observed sometimes in the developmental period [1]. It is also significant to distinguish it from other disturbances of a subclinical nature, while putting the diagnosis of OCD as well as the level of patient functioning and the level of distress $[2,3,4]$.

Evaluation of the frequency of OCD is related to the criteria used. If DSM-IV TR is taken into account they state that OCD appears in about $1-3 \%$ of the population $[5,6]$. On the other hand, diagnosis based upon DSM-5 leads to the conclusion that OCD occurs in $1.1 \%-1.8 \%$ of the population, and is higher in adult females $[7,8]$. In males, it usually appears in childhood $[9,10]$. The authors agree that OCD can be observed in all countries and cultures [3-11].
As a rule, the onset of symptoms is observed during childhood or adolescence and leads to serious deterioration in social and vocational functioning. According to the WHO, OCD was one of the 10 most prominent medical or psychical reasons of disability observed in 1998 in developed countries. Despite the use of the most effective medical and behavioural treatment, it was stated that the symptoms still prevailed in $10 \%$ of patients with OCD $[7,11]$.

Recently, OCD has been considered as a dysfunction of the frontal-basal ganglia thalamo-cortical circuit, which includes the orbito-frontal cortex, the anterior cingulate cortex as cortical parts [7]. In contrast, in ADHD and schizophrenia the dysfunction is associated with hypo-frontality, while OCD is associated with hyper-frontality (fMRI studies) [11]. The group ERP results are inconsistent, which might be a result of heterogeneity in the OCD group.

The current study presents ERP data indicating hyperfrontality in an OCD patient, and aims to evaluate the hyperactivity of the frontal lobes of the patient. 


\section{CASE STUDY}

Since 2008, the 21-year-old female patient had suffered from the presence of obsessions and compulsions from 2008. She was afraid to get up in the mornings, covered her head with a blanket, and finally, in reaction to the long persistent pleas of her mother, she would get up. New difficulties appeared during dressing, which often took up to four hours because she would dress and undress herself several times. Washing was another problem and lasted from 20 minutes to an hour. Moreover, she would wash her hand repeatedly up to 50 times a day with the result that the skin of her hands was sore. She also found it very difficult to leave and re-enter the house, and it took her two before to decide what to do it. Getting undressed was also a complex ritual, for example, after taking off her shoes she wanted the laces to lie in a certain way. If they did not, she would put her shoes on again and take them off until the laces were in the right order. The next activity was touching the bookshelves in the main room while swallowing saliva at the same time. After doing this, she used to go to her room and repeat the same ritual. She was afraid of some objects in the flat and would avoid them. At the same time, she avoided sitting on the chair and sofa, and believed the floor to be safer. In time, she started to fear other objects as well, and would not use newly bought dresses or cosmetics.

Most complicated was the ritual of washing her hair. She washed small strands of her hair separately, and if interrupted she started the process from the very beginning. As a result, washing her hair took up to three hours. She was also afraid to use towels, believing them not to be clean enough and used toilet paper instead. In the evenings, she stood for hours in front of the bed before lying down. At nights, she used to go out into the corridor to keep away bad thoughts. Cycles of intensification and decrease in her disorders could be observed. The intensification was connected with high excitement and fits of rage, which could be provoked by any remark. Somatic problems also appeared, manifesting themselves mainly as head and stomach aches. Depending on the reaction of those closest to her she either calmed down or displayed self-aggression. Each cycle was accompanied with the appearance of a new ear-ring. At present, she has 28-14 rings in each ear.

To sum up, she met the diagnostic criteria of DSM- 5 since her behaviour was characterized by preoccupations on and by repetitive behaviors in response to the preoccupations, despite attempts to decrease or stop those behaviours.

Therapy. Psychodynamic therapy lasting three years did not bring any improvement and her fear of contamination increased. She was reluctant to shake hands, and did not allow herself to be touched even with one finger. For that reason, performing an EEG examination proved to be impossible. Before sitting down she used to put a piece of paper towel on the chair or the bench.

Starting from September 2011, the patient participated in cognitive-behavioural and positive therapy for the next three years. A considerable improvement was noted, but she still was afraid to perform certain actions despite a lowering of the anxiety level. In January 2013, taking a shower took her only 12 minutes, and this encompassed the whole body! Her parents were very happy to see that she had used an ordinary towel. Her recovery was progressing and finally, in May 2014, she agreed to take part both in aneurophysiological and neuropsychological examinations.

EEG recording. The electroencephalogram (EEG) was recorded with the Mitsar 21-channel EEG system (Mitsar, Ltd., http://www.mitsarmedical. com), with a 19-channel electrode cap with tin electrodes that included $\mathrm{Fz}, \mathrm{Cz}, \mathrm{Pz}$, Fp1/2, F3/4, F7/8, T3/4, T5/6, C3/4, P3/4, O1/2. The cap (Electro-cap) was placed on the scalp according to the standard 10-20 system. Electrodes were referenced to linked earlobes (off-line) and the input signals sampled at a rate of $250 \mathrm{~Hz}$ (bandpass $0.5-30 \mathrm{~Hz}$ ). The ground electrode was on the forehead. Impedance was kept below $5 \mathrm{k} \Omega$. The patient sat upright in a comfortable chair looking at a computer screen (17 inches) 1.5 meter in front of her. All recordings were made by the author of this article. ERP waveforms were averaged and computed off-line, and trials with omission and commission errors were automatically excluded $[7,11]$.

Behavioural task. The task consisted of 400 trials sequentially presented to the subject every three seconds. Three categories of visual stimuli were used:

1) 20 different images of animals - referred to later as A;

2) 20 different images of plants - P;

3) 20 different images of people of different professions (presented together with an artificial 'novel' sound), referred to as $\mathrm{H}$.

The trials consisted of presentations of pairs of stimuli with inter-stimulus intervals of $1 \mathrm{~s}$. The duration of stimuli presentation was $100 \mathrm{~ms}$. Four categories of trials were used: A-A, A-P, P-P, and P-H (Fig. 1). In the trials with A-A and $\mathrm{P}-\mathrm{P}$ pairs, the first and the second stimuli were identical (physically the same). The trials were grouped into four sessions with 100 trials in each. In each session a unique set of five A stimuli, five $\mathrm{P}$ and five $\mathrm{H}$ stimuli was selected. Each session consisted of a pseudo-random presentation of 100 pairs of stimuli, with an equal probability for each category and each trial category. The task was to press a button with the right hand to all $\mathrm{A}$-A pairs as fast as possibile, and to withhold from pressing in response to other pairs. The participant performed 10 trials without recording to see if they understood the instruction. She rested for a few minutes after completing 100 trials. Stimuli occupied about $3.8^{\circ}$ of the visual field around the centre of the screen. Visual stimuli (were selected to have) had similar 2D sizes and luminosities.

Artifact correction. Eye blink artifacts were corrected by zeroing the activation curves of individual independent components corresponding to eye blinks. These components were obtained by the application of Independent Component Analysis (ICA) to the raw EEG fragments, as described in Vigário [12] and Jung et al. [13]. Epochs with excessive amplitude of filtered EEG and/or excessive faster and/or slower frequency activity were automatically marked and excluded from further analysis. The exclusion thresholds were set as follows:

1) $100 \mu \mathrm{V}$ for non-filtered $\mathrm{EEG}$;

2) $50 \mu \mathrm{V}$ for slow waves in $0-1 \mathrm{~Hz}$ band;

3) $35 \mu \mathrm{V}$ for fast waves filtered in the band $20-35 \mathrm{~Hz}$.

In addition, the recordings and excluded remaining artifacts we visually inspected. EEG spectra were computed for Eyes Open, Eyes Closed and the GO/NOGO task 
conditions separately. The artifact free fragments of EEG were divided into $4 \mathrm{sec}$ epochs with $50 \%$ overlap. The Hanning time window was used [14]. The EEG spectra were computed for each epoch and averaged. The Mean value and standard deviations for each $0.25 \mathrm{~Hz}$ bin were computed. For comparison, EEG spectra pre and before after intervention the t-test was used.

\section{RESULTS}

The results of comparison in the behavioural parameters of the patient with the average data of the group of healthy subjects $(\mathrm{N}=57)$ of the same age are presented in Table 1.

Table 1. Behavioural data in the cued GO/NOGO task

\begin{tabular}{lcccc}
\hline Data & $\begin{array}{c}\text { Omission } \\
\text { errors }\end{array}$ & $\begin{array}{c}\text { Commission } \\
\text { errors }\end{array}$ & $\begin{array}{c}\text { Reaction time } \\
\text { (RT) in ms }\end{array}$ & $\begin{array}{c}\text { Error of the RT } \\
\text { Variance in ms }\end{array}$ \\
\hline Patient & $3 \%$ & $8 \%$ & 275 & 5.2 \\
\hline Healthy controls & $1.9 \%$ & $0.8 \%$ & 408 & 8.6 \\
\hline p-value & 0.7 & 0.00 & 0.18 & 0.21 \\
\hline
\end{tabular}

The subject is significantly impulsive. The reaction time is much faster than the mean of the healthy control group by $130 \mathrm{~ms}$, but does not reach a statistically significant level of confidence.

EEG spectra. In the EEG spectra in all three conditions (EO, EC, GO/NOGO task), the two characteristics were deviant from the normative average data; they were: an increase of beta activity frontally and an increase of alpha activity parietally. The two independent components corresponding to these deviations from the reference are presented in Fig. 1. The independent components were extracted by Infomax algorithm (WinEEG software by Mitsar, Ltd) from spontaneous EEG in the eyes closed condition.

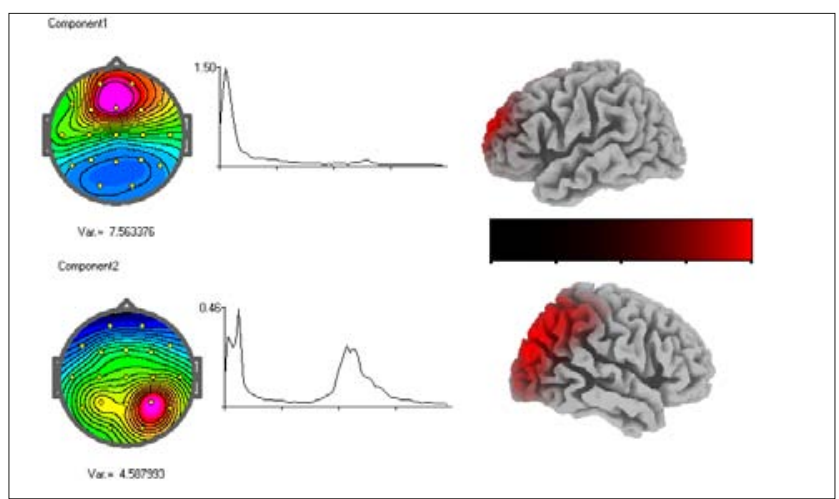

Figure 1. Independent components corresponding to deviations from the reference

a) topography, spectra and sLORETA image of the frontal component.

b) topography, spectra and SLORETA image of the right parietal component.

Event related potentials in the cued GO/NOGO task. The results of a comparison of the patient's ERPs in the three conditions: Continue (1 stimulus), NOGO and GO (2 stimulus) are presented in Fig 2. As can be seen, the ERPs show an increase of the components elicited in these conditions: CNV (Fig. 2A), P3 GO (Fig. 2B) and P3 NOGO (Fig. 2C). All components show elevations above the average components, with maximums around $\mathrm{Cz}$ (see the maps of the deviations from the reference). The sLORETA images show the sources of the differences within the frontal-parietal networks.

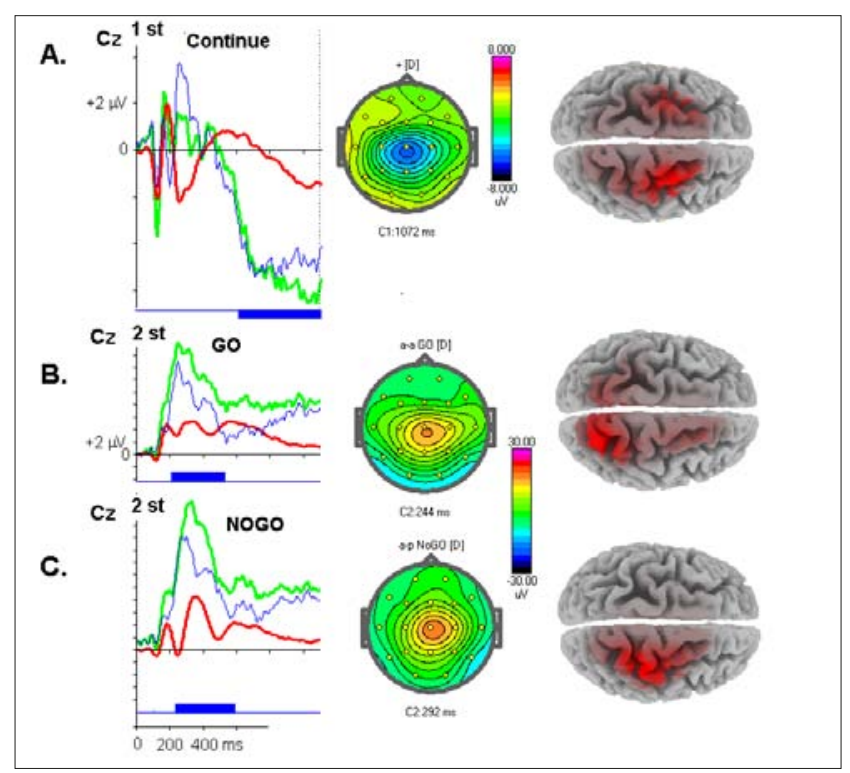

Figure 2. ERP deviations from the norms

$A$ - ERP in continued trials. From left to right - ERPs recorded at $\mathrm{Cz}$ for the patient (green), the averaged group of healthy subjects (red), and the difference wave as patient-healthy (blue). Blue box below indicates $p<0.01$, the map at maximum of the difference wave, sLORETA image of the difference wave.

$B$ - ERP in GO trials. From left to right - ERPs recorded at Cz for the patient (green), the averaged group of healthy subjects (red) and the difference wave as patienthealthy (blue). The blue box below indicates $p<0.01$, the map at maximum of the difference wave, sLORETA image of the difference wave..

$\mathrm{C}$ - ERPs in NOGO trials. From left to right - ERPs recorded at $\mathrm{Cz}$ for the patient (green), the averaged group of healthy subjects (red), and the difference wave as patient-healthy (blue). Blue box below indicates $p<0.01$, the map at maximum of the difference wave, sLORETA image of the difference wave.

\section{DISCUSSION}

Recent studies show that the persistence of obsessions and compulsions may result from disturbances in the functioning of several processes. The first is connected with a strong urge to perform a given action. For example, washing hands because the patient feels that they are contaminated. Even images of dirty hands may provoke strong emotional reactions in OCD patients due to the dysfunction of the emotional system [11]. The next process is the ability to stop performing the action, when the patient feels that everything is alright. For example, the patient knows that her hands are clean since she has just washed them. That ability depends upon the work of the executive system [12]. Lack of coordination between the emotional and executive systems may also result in the occurrence of OCD symptoms. Since patients with OCD make significantly more commission mistakes than controls in GO/NOGO tasks, it is highly probable that they have lost the ability to inhibit the ongoing action. They seem unable to monitor their actions and to evaluate the discrepancy between the planned action and the actual outcome. Hence, they are unable to change their behaviour.

Another important process connected with the executive system is an ability to change an ongoing action. Yet, the results of experiments with the Card Sorting Test, which included patients with OCD, were far from uniquivocal. Some authors observed the occurrence of persistence in OCD 
and others did not report such disorders. At the root of OCD symptoms, the hyperactivity of the neuronal circle including caudate nucleus, which creates the urge 'to do something', the orbital prefrontal cortex, which creates the feeling that 'something is not OK', and the cingulate gyrus cortex, which makes the patient feel uncertain and embarrassed [8]. It is also connected with the emotional circle, including the orbital frontal cortex, the ventral part of the anterior cingulate gyrus, and the caudate nucleus. In addition, it is connected with the pre-supplementary motor cortex and the dorsal part of the anterior cingulate cortex, as well as the putamen and caudate nucleus, which is closely connected with the motor system. This leads to an enhancement of the action monitoring operations [11].

The presented study shows that the higher the verbal activity of the patient, the lower the level of her disorders. This means that a great number of social contacts resulted in improvement in her behaviour. According to the microgenetic theory, developing new strategies (e.g., sitting down directly on the chair without first placing a paper towel on it) makes possible a 'de-blocking' of neuronal connections $[15,16,17$, 18]. This increases the integrity of the white matter in the subcortical structures, ventral premotor cortex, posterior part of the corpus callosum, left angular gyrus, and the cerebellum $[7,11]$, resulting in the gradual decrease in the symptoms of OCD. Further confirmation of this assumption can be found in studies which report a decrease in OCD symptoms with age [11]. The mechanism of the decrease is probably the same in both cases [14]. New strategies acquired during the process of therapy may also be used in everyday situations, and make possible the 'de-blocking' of neuronal connections $[8,11,15]$ which leads to the decrease of obsessive-compulsive behaviours.

\section{CONCLUSIONS}

Specific patterns of QEEG and ERPs, the increase of beta activity frontally, and the increase in the parietal alpha activity pattern which produces hyper-frontality, might be useful in the diagnosis of OCD patients. ERPs in a GO/ NOGO task can be also used in the assessment of functional brain changes in these patients.

\section{REFERENCES}

1. Diagnostic and statistical manual of mental disorders: DSM-5 (5 ed.). Washington: American Psychiatric Publishing. 2013. pp. 237-242.

2. Fenske JN, Schwenk TL. Obsessive compulsive disorder: diagnosis and management". Am Fam Physician. 2009; 80 (3): 239-45.

3. Grant JE. Clinical practice: Obsessive-compulsive disorder". N Eng J Med. 2014; 371 (7): 646-53.

4. Goodman WK, Grice DE, Lapidus KA, Coffey BJ. Obsessive-compulsive disorder. Psychiatr Clin North Am. 2014; 37 (3): 257-67.

5. Koran LM. Obsessive-Compulsive Disorder: An Update for the Clinician. Focus 2007;(5): 3.

6. Abramowitz JS, Taylor S, McKay D.Obsessive-compulsive disorder. Lancet 2009; 374 (9688): 491-9.

7. Kropotov JD. Quantitative EEG, event-related potentials and neurotherapy. Amsterdam, London: Elsevier Academic Press. 2009.

8. Pąchalska M, Kaczmarek BLJ, Kropotov JD. Neuropsychologia kliniczna: od teorii do praktyki. Warszawa: Wydawnictwo Naukowe PWN. 2014.

9. Shin NY, Lee TY, Kim E, Kwon JS. Cognitive functioning in obsessivecompulsive disorder: a meta-analysis. Psychol Med. 2013; 44: 1-10.

10. Kolb B, Whishaw I. Fundamentals of human neuropsychology. $6^{\text {th }}$ Ed., New York: Worth Publishers. 2007.

11. Kropotov JD. Functional neuromarkers for psychiatry. Elsevier Academic Press. 2016.

12. Góral-Półrola J, Żychowska M, Ziółkowski A., Pąchalska M. Changes and expression of selected genes associated with cell stress in chronic stuttering: Case study. Acta Neuropsychol. 2015; 13(4): 390-401.

13. Pachalska M, Góral-Półrola J, Brown JW, MacQueen BD.Consciousness and reality: a neuropsychological perspective. Acta Neuropsychol. 2015; 13(3): 205-227.

14. Menzies L, Achard S, Chamberlain SR, Fineberg N, Chen CH, del Campo N, Sahakian BJ, Robbins TW, Bullmore E. Neurocognitive endophenotypes of obsessive-compulsive disorder. Brain. 2007; 130 (Pt 12): $3223-36$.

15. Starcevic V, Janca A. Obsessive-compulsive spectrum disorders: still in search of the concept-affirming boundaries. Current opinion in psychiatry. 2011; 24 (1): 55-60.

16. Kropotov J D, Ponomarev V A, Kropotova O V, Anichkov A D, Nechaev $\mathrm{V}$ B. Human auditory-cortex mechanisms of preattentive sound discrimination. Neurosci Lett. 2000; 280: 87-90.

17. Markarian Y, Larson MJ, Aldea MA, Baldwin SA, Good D, Berkeljon A, Murphy TK, Storch EA, McKay D. Multiple pathways to functional impairment in obsessive-compulsive disorder". Clin Psychol Rev. 2010; 30 (1): $78-88$.

18. Mirski A, Pąchalska M, Moskała M, Orski M, Orska M, Miąskiewicz M, Zapała J, Kropotov JD. Neuromarkers of anxiety and depression in a patient after neuro-ophthalmic surgery of the meningioma - effect of individually-tailored tDCS and neurofeedback. Ann Agric Environ Med. 2015; 22(4): 730-735. 